\title{
The Sting of Rejection: Deferring Blood Donors due to Low Hemoglobin Values Reduces Future Returns
}

\author{
Adrian Bruhin $^{a} \quad$ Lorenz Goette $^{\mathrm{b}, \mathrm{c}} \quad$ Simon Haenni ${ }^{\mathrm{d}} \quad$ Lingqing Jiang $^{\mathrm{e}}$ \\ Alexander Markovic $^{f} \quad$ Adrian Roethlisberger $^{f} \quad$ Regula Buchlif $^{f}$ Beat M. Frey ${ }^{f}$ \\ ${ }^{a}$ Faculty of Business and Economics (HEC Lausanne), University of Lausanne, Lausanne, Switzerland; ${ }^{\text {b}}$ Department

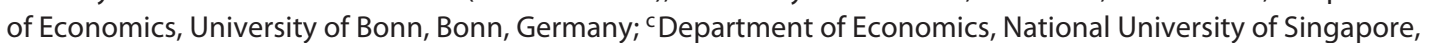 \\ Singapore, Singapore; ${ }^{d}$ Department of Economics, University of Zurich, Zurich, Switzerland; ${ }^{e}$ Department of \\ Economics, University of Essex, Colchester, UK; ' Blood Transfusion Service, Swiss Red Cross, Schlieren, Switzerland
}

\section{Keywords}

Blood donation · Donor deferral · Donor return .

Quasi-experiment

\begin{abstract}
Background: Roughly one quarter of short-term temporary deferrals (STTD) of blood donors are low-hemoglobin deferrals (LHD), i.e. STTD due to a hemoglobin $(\mathrm{Hb})$ value falling below a cutoff of $125 \mathrm{~g} / \mathrm{L}$ for female and $135 \mathrm{~g} / \mathrm{L}$ for male donors. Since voluntarily donating blood is a prosocial activity, donors may perceive deferral as social exclusion, which can cause social pain, decrease self-esteem, and lead to antisocial behavior. However, little is known about the causal impacts of LHD on donor return. Study Design and Methods: We conducted a quasi-experiment with 80,060 donors invited to blood drives in the canton of Zurich, Switzerland, between 2009 and 2014. Within a narrow window of Hb values around the predetermined cutoff, the rate of LHD jumps discontinuously. This discontinuous jump allows us to quantify the causal effects of LHD on donor return, as it is uncorrelated with other unobserved factors that may also affect donor return. Results: We found different behavioral reactions to LHD for female and male donors. Female donors do not react to the first LHD. However, after any repeated LHD, they are 13.53 percentage points $(p<0.001)$ less likely to make at least 1 donation attempt within the next 18 months
\end{abstract}


Fig. 1. Procedure of the BTSRC for measuring and recording $\mathrm{Hb}$ values. To reduce the rate of false LHD, the BTSRC applies a specific procedure for recording $\mathrm{Hb}$ values. When the first measured $\mathrm{Hb}$ value is above the cutoff, the staff records that value and clears the individual for donation. However, when the first measurement falls below the cutoff, the staff repeats the measurement twice and records the average $\mathrm{Hb}$ value over all 3 measurements. Only if the final 2 measurements are both above the cutoff does the staff clear the individual for donation.

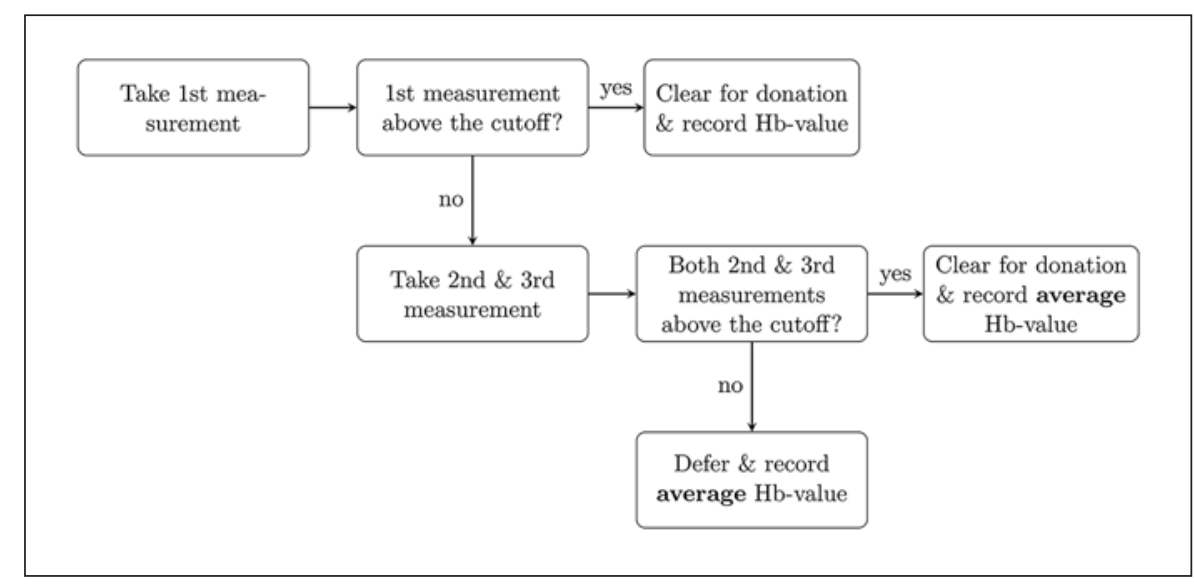

leads to false LHD where the donors' true $\mathrm{Hb}$ value is above the cutoff [4]. While most studies on donor return have focused on the effects of material incentives [5-8], and on correlations with donor characteristics [9], little is known about the causal impact of LHD on donor return.

Donors may take LHD as an excuse to refrain from donating blood in the future. In line with the literature on excuse-driven behavior $[10,11]$, donors who experience an LHD may believe that being deferred again is likely and thus refrain from future donation attempts. Furthermore, donors may perceive LHD as social exclusion. Studies on other types of prosocial activities have suggested that social exclusion can cause social pain and decrease self-esteem, even if it is only implicit and due to exogenous circumstances rather than the behavior of others [12-15].

The way in which individuals respond to social exclusion is ambiguous. On the one hand, individuals may respond to exclusion in socially desirable ways to satisfy their need for belongingness, but on the other hand they may also exhibit antisocial reactions if they have no control over the causes of their exclusion [16]. As donors cannot control their $\mathrm{Hb}$ values at a given point in time, we hypothesize that deferred donors may refrain from future donation attempts or, at least, reduce their donation frequency. A study examining the effects of various shortterm temporary deferrals (STTD) by comparing donation rates of donors, computer matched based on age, gender, and donation date supports this hypothesis. It found that donors who experienced an LHD were 13.6\% less likely to return over the next 4.25 years than nondeferred donors [17]. More recent studies have also reported negative correlations between STTD and future donation attempts [18-21]. However, deferred and nondeferred donors may differ, for example, in terms of their health status, which may also affect donor return. This makes the interpretation of these correlational results, and in particular of their magnitudes, difficult. We provide a novel causal analysis that identifies the local average treatment effect of LHD on donor return among otherwise identical marginal donors.

Repeated LHD might even have a stronger detrimental effect on donor return. Prior evidence shows that repeated social exclusion can lead to maladaptive responses causing further deferrals [22] and depression [23]. Since we only observed donation attempts in a given period, we differentiate between the first LHD and repeated LHD during our study period. We quantify the causal effect of LHD on donor return both overall and for repeated LHD.

\section{Materials and Methods}

\section{Empirical Setup}

Our sample comprised 80,060 voluntary blood donors who had donated at least once before this study. They were repeatedly invited to blood drives organized by the Blood Transfusion Service of the Red Cross in Zurich, Switzerland (BTSRC). Blood drives typically take place twice a year at the same location.

We observed 260,026 donation attempts during the sample period from January 2009 to November 2014. For each donation attempt, we also observed the donors' gender, age, blood type, recorded $\mathrm{Hb}$ value, and whether the donation attempt failed due to an LHD or some other reason. The term "donation attempt" refers to both successful and failed donations, as failed donations equally show the willingness to donate. Our data set comprised no further information.

The BTSRC applies different Hb cutoffs for female ( $\geq 125 \mathrm{~g} / \mathrm{L})$ and male $(\geq 135 \mathrm{~g} / \mathrm{L})$ donors and may give gender-specific feedback in response to LHD. Therefore, we analyzed female and male donors separately.

In total, $42.9 \%$ of the donors were female, accounting for $39.1 \%$ of all donation attempts (online suppl. Table S1 for detailed descriptive statistics; for all online suppl. material, see www.karger. $\mathrm{com} /$ doi/10.1159/000500679). The mean $( \pm \mathrm{SD})$ age of the female donors was $39.73( \pm 14.00)$ years, and that of the male donors was $42.65( \pm 13.82)$ years. The mean $\mathrm{Hb}$ value was $137.71( \pm 10.47) \mathrm{g} / \mathrm{L}$ for female donors and $153.21( \pm 11.17) \mathrm{g} / \mathrm{L}$ for male donors. 




(a) female

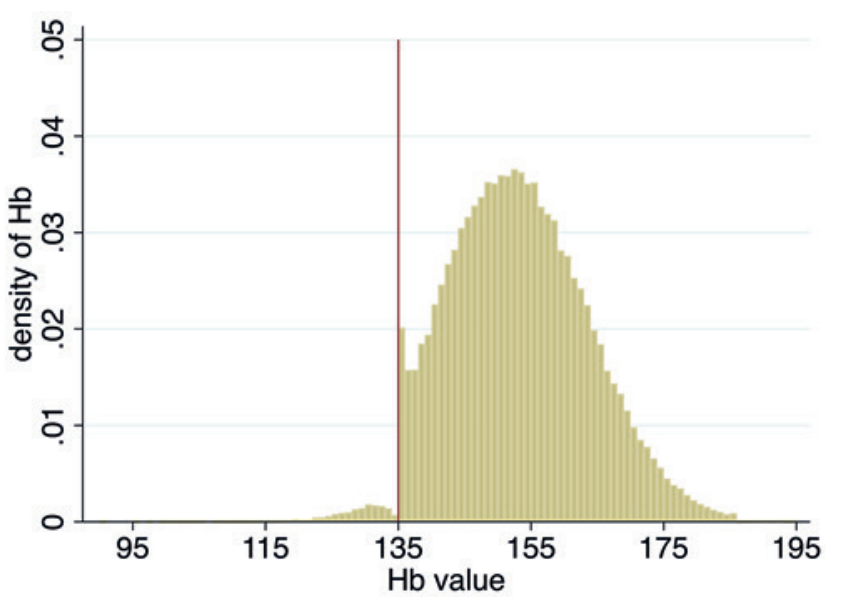

(b) male

Fig. 2. Distribution of recorded $\mathrm{Hb}$ values $(\mathrm{g} / \mathrm{L})$. The normal distribution of the recorded $\mathrm{Hb}$ values at the cutoff is distorted because $\mathrm{Hb}$ values just below the cutoff are more likely to be revised (Fig. 1; Table 1). However, since the distortion originates directly from the procedure of the BTSRC and not from the donors' behavior, it is uncorrelated with donor return. Thus, LHD around the cutoff are exogenous with respect to donor return.

\section{Low-Hemoglobin Deferrals}

In total, 12.4 and $8.1 \%$ of donation attempts by female and male donors, respectively, were deferred for any reason. About $4.7 \%$ of the donation attempts by female donors (38\% of all deferrals) and $1.2 \%$ of the attempts by male donors ( $15 \%$ of all deferrals) resulted in LHD. Female donors are more likely to experience LHD, as their cutoff is stricter relative to their baseline $\mathrm{Hb}$ values and their $\mathrm{Hb}$ values tend to vary more due to the menstruation cycle. LHD can occur repeatedly; $1.3 \%$ of the female donors and $0.4 \%$ of the male donors experienced more than 1 LHD.

There is substantial error in the measurement of $\mathrm{Hb}$ values, mainly due to imprecise mobile measurement devices $[1,4,24]$. To avoid false LHD, the BTSRC applies the procedure illustrated in Figure 1 for recording $\mathrm{Hb}$ values. When the first measured $\mathrm{Hb}$ value is above the cutoff, the staff records it and clears the individual for donation. However, when the first measurement falls below the cutoff, the staff repeats the measurement 2 more times and records the average value of all 3 measurements. Only if the final 2 measurements are both above the cutoff, the staff clears the individual for donation.

Table 1 presents possible scenarios that may arise under this procedure. For instance, a recorded $\mathrm{Hb}$ value of $124 \mathrm{~g} / \mathrm{L}$ for a female donor may lead to opposite outcomes. In scenario a, an insufficient $\mathrm{Hb}$ value is followed by 2 sufficient $\mathrm{Hb}$ values, resulting in a successful donation attempt. In scenario b, an insufficient $\mathrm{Hb}$ value is followed by 1 sufficient and 1 insufficient $\mathrm{Hb}$ value, resulting in an LHD. Similarly, when we observe a recorded $\mathrm{Hb}$ value of $125 \mathrm{~g} / \mathrm{L}$, it can either be the first and only measurement, directly leading to a successful donation, or it can be the average of 3 measurements, 2 of which are insufficient, leading to an LHD.

The procedure has 2 consequences. First, it introduces noise around the cutoff which makes the recorded $\mathrm{Hb}$ value a fuzzy, i.e., probabilistic, indicator for LHD. Second, as visualized in Figure 2, it results in a distorted normal distribution of the recorded $\mathrm{Hb}$ values at the cutoff because $\mathrm{Hb}$ values just below the cutoff are revised
Table 1. Example of scenarios leading to different recorded $\mathrm{Hb}$ values and outcomes

\begin{tabular}{|c|c|c|c|c|c|}
\hline \multirow[t]{2}{*}{ Scenario } & \multicolumn{3}{|c|}{ Hb measurement, g/L } & \multirow{2}{*}{$\begin{array}{l}\text { Recorded average } \\
\mathrm{Hb} \text { value, g/L }\end{array}$} & \multirow[t]{2}{*}{ Outcome } \\
\hline & $1 \mathrm{st}$ & 2nd & $3 \mathrm{rd}$ & & \\
\hline $\mathrm{a}$ & 120 & 125 & 127 & 124 & success \\
\hline $\mathrm{b}$ & 124 & 125 & 123 & 124 & LHD \\
\hline $\mathrm{C}$ & 125 & - & - & 125 & success \\
\hline $\mathrm{d}$ & 124 & 130 & 121 & 125 & LHD \\
\hline
\end{tabular}

All examples are for female donors with a cutoff at $\mathrm{Hb} \geq 125$ $\mathrm{g} / \mathrm{L}$. A recorded $\mathrm{Hb}$ value of $124 \mathrm{~g} / \mathrm{L}$ can originate from 2 different scenarios with opposite outcomes. In scenario a, an insufficient $\mathrm{Hb}$ value is followed by 2 sufficient $\mathrm{Hb}$ values, resulting in a successful donation attempt. In scenario $\mathrm{b}$, an insufficient $\mathrm{Hb}$ value is followed by 1 sufficient and 1 insufficient $\mathrm{Hb}$ value, resulting in an LHD. Similarly, when we observe a recorded $\mathrm{Hb}$ value of $125 \mathrm{~g} / \mathrm{L}$, it can either be the first and only measurement, directly leading to a successful donation (scenario c), or it can be the average of 3 measurements, 2 of which are insufficient, leading to an LHD (scenario d).

more often. However, since this distortion originates directly from the BTSRC procedure and not from the donors' behavior, it is uncorrelated with donor return. Hence, given that the donors cannot manipulate their $\mathrm{Hb}$ values at a given point in time, LHD around the cutoff are exogenous to donor return.

In case of an LHD, the BTSRC communicates the temporary nature of the shortfall in the $\mathrm{Hb}$ value and recommends that the donor wait 3 months before making the next donation attempt. This corresponds to the waiting period after a successful donation. 


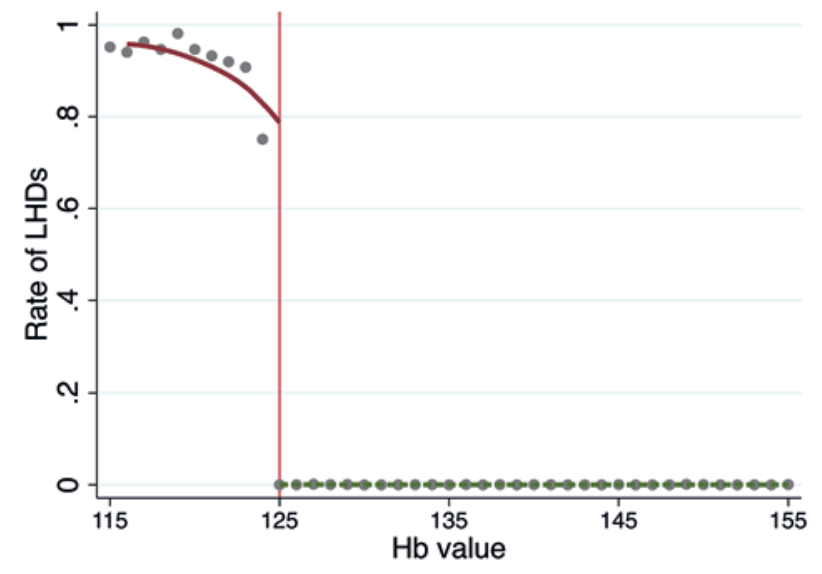

(a) Female

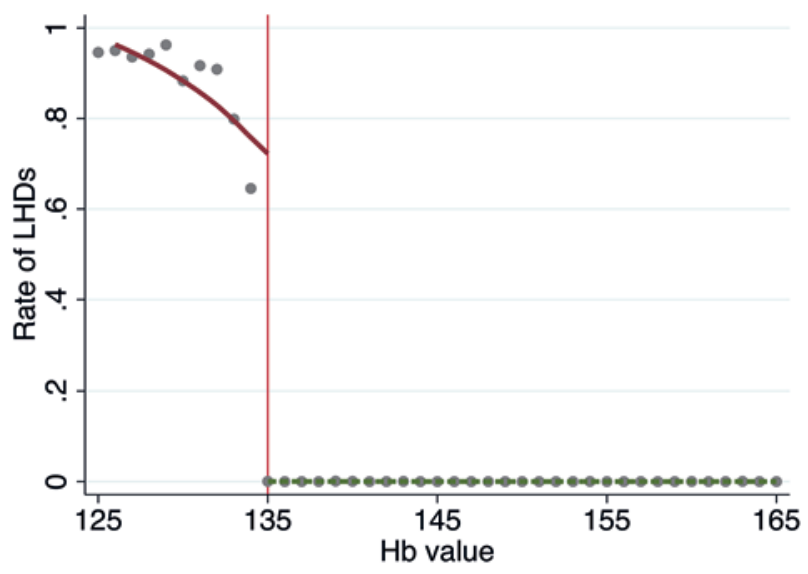

(b) Male

Fig. 3. Rate of $\mathrm{LHD}$ at each recorded $\mathrm{Hb}$ measurement. For female donors the cutoff is $\mathrm{Hb}<125 \mathrm{~g} / \mathrm{L}$ and for male donors it is $\mathrm{Hb}<135 \mathrm{~g} / \mathrm{L}$. The rate of donors experiencing an LHD jumps discontinuously at the cutoff; donors do not experience any LHD when their $\mathrm{Hb}$ value is above the cutoff but this rate increases discontinuously once their $\mathrm{Hb}$ value falls below the cutoff.

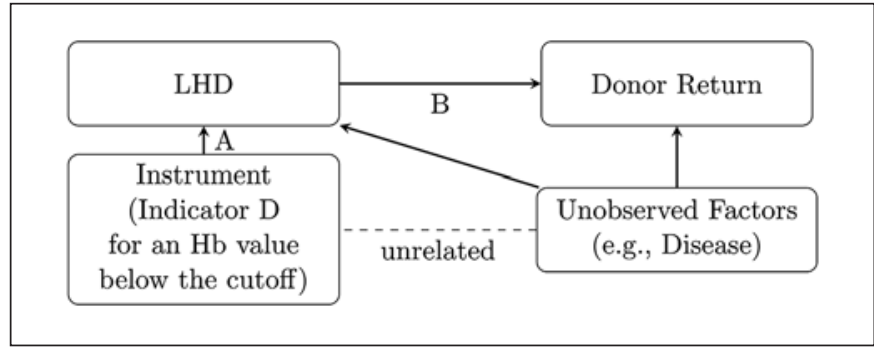

Fig. 4. Empirical strategy using an instrument to quantify the causal effect of LHD on donor return. The indicator D whether a donor's $\mathrm{Hb}$ value is below the cutoff serves as an instrument for quantifying the effect of LHD on the donor's return. The indicator D satisfies 2 properties, making it a strong and valid instrument. First, D has a strong effect on the probability of experiencing an LHD (Fig. 5). Second, at the margin, D is exogenous with respect to all other unobserved factors that may be related to both the probability of experiencing an LHD and the donor's return rate. Hence, the variation in LHD caused by D is exogenous with respect to all unobserved factors (arrow in A) and can be used to quantify the causal effect of LHD on donor return (arrow in B).

\section{Donor Return}

We defined donor return in the following 2 ways: (1) as an indicator of whether a donor makes at least 1 donation attempt within the next 18 months after the last donation attempt, and (2) as the number of donation attempts within the next 18 months after the last donation attempt. The first measure indicates whether the donors stay in the pool at all after experiencing a single or repeated LHD. The second measure tells us how they adjust their donation frequency after a single or repeated LHD. We dropped the last 18 months of our data set, because for any potential LHD during this period there was not enough time to observe the donors' future donation attempts. The resulting sample size was 29,371 female donors with 77,170 donation attempts and 40,145 male donors with 119,658 donation attempts.

In total, $65.5 \%( \pm 47.5)$ of the female donors and $71.7 \%( \pm 45.0)$ of the male donors made at least 1 donation attempt within the next 18 months. On average, female donors made $1.200( \pm 1.133)$ and male donors made $1.442( \pm 1.222)$ donation attempts within the next 18 months.

\section{Quantifying Causal Effects of LHD on Donor Return}

We used a regression discontinuity (RD) design to quantify the causal effects of LHD on donor return [25]. The RD design exploits the fact that the BTSRC follows the cutoff in $\mathrm{Hb}$ values for accepting or deferring donors. It compares the return of donors whose $\mathrm{Hb}$ values are just above and just below the cutoff within a narrow window of -10 and $+30 \mathrm{~g} / \mathrm{L} \mathrm{Hb}$ around the cutoff. (Results based on an alternative, symmetric window of -10 and $+10 \mathrm{~g} / \mathrm{L} \mathrm{Hb}$ around the cutoff can be found in in the online suppl. material.)

Within this narrow window, donors above and below the cutoff differ only slightly in their $\mathrm{Hb}$ values. Thus, any unobserved factors related to both the $\mathrm{Hb}$ values and the donors' return - such as deceases - are comparable for donors with $\mathrm{Hb}$ values just above and below the cutoff. However, the probability of experiencing an LHD jumps discontinuously at the cutoff; donors with $\mathrm{Hb}$ values just below the cutoff are deferred much more frequently than those with $\mathrm{Hb}$ values just above the cutoff. Consequently, any difference in their return is due to the discontinuous jump in the probability of experiencing an LHD.

Figure 3 illustrates that the rate of donors experiencing an LHD in fact jumps discontinuously at the cutoff. Both female (Fig. 3a) and male (Fig. 3b) donors did not experience any LHD when their $\mathrm{Hb}$ values were above the cutoff. However, once their $\mathrm{Hb}$ values fell below the cutoff, the rate of LHD increased discontinuously. At the same time, as can be seen in the online supplementary material, none of the donors' other observable characteristics (except for having the blood type $\mathrm{O}-$, which is in especially high demand 


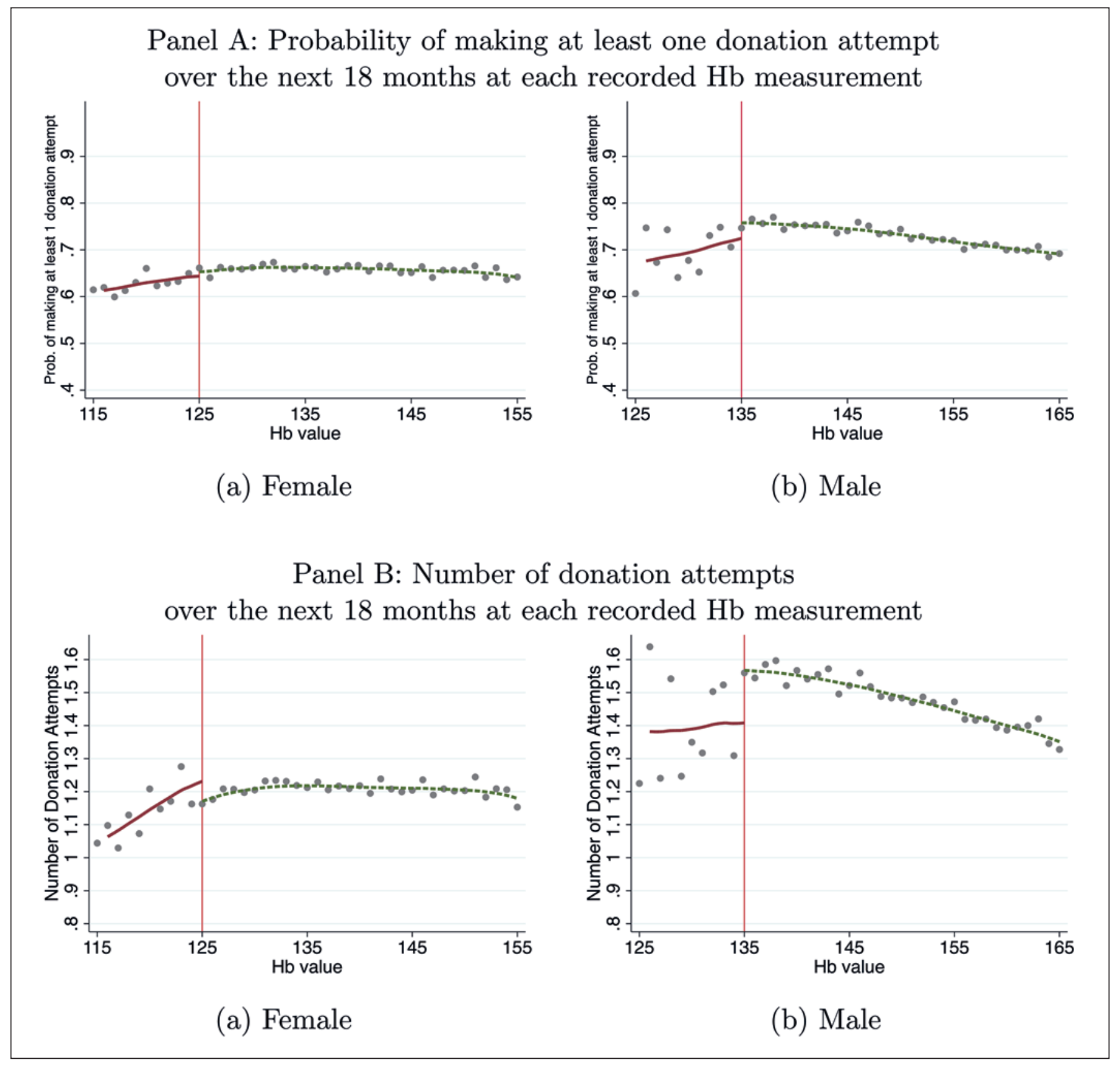

Fig. 5. Donor return by $\mathrm{Hb}$ measurement. The red and green lines are based on local linear regressions. The dots represent the mean probabilities $(\mathbf{A})$ and numbers $(\mathbf{B})$ by $\mathrm{Hb}$ value.

and may be subject to different regulations) jumps at the cutoff. This confirms that donors with $\mathrm{Hb}$ values just below and above the cutoff differ exclusively in the probability of experiencing LHD.

However, not all donors with an $\mathrm{Hb}$ value below the cutoff experience LHD. Figure 3 reveals that the rate of LHD is about $80 \%$ for female donors and $75 \%$ for male donors whose $\mathrm{Hb}$ values are just slightly below the cutoff. This follows from the procedure the BTSRC applies for mitigating false LHD, which implies that some donors with recorded $\mathrm{Hb}$ values slightly below the cutoff can still donate (Fig. 1; Table 1). Formally, this means that $\mathrm{Hb}$ values below the cutoff are fuzzy indicators of LHD.

To take this fuzziness into account, we had to use a so-called fuzzy RD design. In the fuzzy RD design, the indicator $\mathrm{D}$ of wheth- er a donor's $\mathrm{Hb}$ value is below the cutoff serves as an instrument for quantifying the effect of LHD on the return rate. As shown in Figure 4, the indicator D satisfies 2 properties, making it a strong and valid instrument. First, D has a strong effect on the probability of experiencing an LHD, which jumps when the Hb value falls below the cutoff. Second, D is exogenous with respect to all other unobserved factors that may be related to both the probability of experiencing an LHD and the donor's return rate. This is because, within the narrow window around the cutoff, whether the $\mathrm{Hb}$ value is below $(\mathrm{D}=1)$ or above $(\mathrm{D}=0)$ the cutoff is unrelated to any of these other factors. To quantify the effect of LHD on donor return in the fuzzy RD design, we have to apply an instrumental variable estimator [25]. Intuitively, such an estimator proceeds in 2 
stages. First, it estimates the difference in return rates between donors with $\mathrm{Hb}$ values above and below the cutoff. In the second stage, it takes into account the fuzziness of the indicator D and scales up the difference in donor return by the predicted difference in the rate of LHD. As an illustration, consider for example male donors. As can been seen in Figure 5b, male donors with $\mathrm{Hb}$ values just below the cutoff made roughly $1.57-1.40=0.17$ fewer donation attempts within the subsequent 18 months than their peers with $\mathrm{Hb}$ values just above the cutoff. Thus, in the first stage, the estimated difference in donor return was -0.17 . However, Figure 3 shows that only about $75 \%$ of male donors with an $\mathrm{Hb}$ value below the cutoff experienced LHD. Thus, in the second stage, the difference in donor return was scaled up to $-0.17 / 0.75=-0.23$ to estimate by how much LHD reduced the male donors' number of donation attempts within the next 18 months.

The instrumental variable estimator used in our empirical models is called 2-stage least squares. It has a similar intuition but additionally provides standard errors and allows us to include control variables to increase precision. For details, see the online supplementary material.

\section{Empirical Models}

We estimated the effects of LHD on donor return in 2 empirical models. The first model estimates the overall effect of LHD, while the second distinguishes between the effects of the first versus repeated LHD.

In both models, we used the 2 definitions of donor return to specify the outcome variables. In version A of the models, the outcome variable is the probability of making at least 1 donation attempt within the next 18 months, while in version $\mathrm{B}$, it is the number of donation attempts within the next 18 months.

The first model estimates the overall effect of LHD with a single indicator. The second model adds an interaction term between the indicators of the current LHD and past LHD to differentiate between the effects of the first versus repeated LHD. The second model also includes an indicator of past LHD as a control variable to give the interaction term the desired interpretation.

Besides estimating the effects of LHD on donor return, both models include control variables to increase precision. These control variables are the recorded $\mathrm{Hb}$ value and an interaction term between the recorded $\mathrm{Hb}$ value and the indicator $\mathrm{D}$, as well as age specified as a third-degree polynomial. Specifying a third-degree polynomial was necessary, as we expected age to affect donor return nonlinearly, in line with previous research [21]. Both models also include the donors' blood types and month fixed effects to control for permanent blood type-related and seasonal differences in the supply and demand of blood transfusions. For details about the 2 empirical models, see the online supplementary material.

\section{Results}

This section presents descriptive evidence and the estimation results. First, it illustrates how donor return reacts to changes in the Hb value at the cutoffs. Subsequently, it reports the effects of LHD on donor return according to the 2 empirical models.

\section{Descriptive Evidence}

We first show descriptive evidence for the relationship between donor return and the recorded $\mathrm{Hb}$ value.
Figure 5a displays how the probability of making at least 1 donation attempt within the next 18 months reacts to changes in the $\mathrm{Hb}$ value close to the cutoff. For female donors, there is hardly any difference in the probability of making at least 1 donation attempt when their $\mathrm{Hb}$ value is below versus above the cutoff of $125 \mathrm{~g} / \mathrm{L}$. For male donors, there is a small discontinuous decrease in the probability of making at least 1 donation attempt when their $\mathrm{Hb}$ value falls below the cutoff. Figure $5 \mathrm{~b}$ indicates how the average number of donation attempts within the next 18 months reacts to changes in the $\mathrm{Hb}$ value close to the cutoff. As with the other measure of donor return, there is hardly any difference for female donors in the number of donation attempts when their $\mathrm{Hb}$ value falls below the cutoff. For male donors, however, there is a discontinuous decrease when their $\mathrm{Hb}$ value falls below the cutoff.

The discontinuous decrease at the cutoff indicates that LHD negatively affected the male donors' return rate. We now quantify these effects by estimating the empirical models.

\section{The Causal Effects of LHD on Donor Return}

Table 2 shows the results of the first empirical model estimating the overall effect of LHD on donor return. (The first stage regressions can be found in online suppl. Table S2.)

There is a difference between the overall reactions of female and male donors to LHD. The female donors' return rate reacts neither in terms of the probability of making at least 1 donation attempt (point estimate: -0.034 ; $p=0.150)$ nor in terms of the number of donation attempts (point estimate: $0.009 ; p=0.877$ ), as both coefficients are insignificant.

In contrast, LHD strongly affects the male donors' overall return rate. They are 6.07 percentage points $(p=$ $0.095)$ less likely to make at least 1 donation attempt within the next 18 months and also make $0.221(p=0.024)$ fewer donation attempts within the same period. This effect nicely coincides with the crude analysis $(1.57-1.40=$ $0.17)$ described earlier in Materials and Methods. Relating the estimated effect to the baseline number of donation attempts (online suppl. Table S1) reveals that male donors make $0.221 / 1.442=15.33 \%$ fewer donation attempts within the first 18 months after an LHD. The difference between female and male donors is insignificant in version $\mathrm{A}(p=0.578)$ but significant in version $\mathrm{B}(p=$ $0.067)$ of the model.

$\mathrm{The} \mathrm{Hb}$ value itself correlates with donor return only negligibly within the considered window of -10 to $30 \mathrm{~g} / \mathrm{L}$ around the cutoff, although the coefficients are significant. For female donors, a 1-unit increase in the $\mathrm{Hb}$ value above the cutoff is related to a decrease of 0.0931 percentage points in the probability of making at least 1 donation 
Table 2. Overall effects of LHD on donor return

\begin{tabular}{lll}
\hline $\begin{array}{l}\text { Version A } \\
\text { Dependent variable: probability of making at }\end{array}$ & Females & \\
$\begin{array}{l}\text { least } 1 \text { donation attempt within the next } 18 \\
\text { months }\end{array}$ & & Males \\
\hline Mean (dependent variable) & 0.655 & \\
LHD & $-0.0342(0.0238)$ & 0.717 \\
$\mathrm{Hb}$ & $-0.000931^{* * *}(0.000243)$ & $-0.0607^{*}(0.0364)$ \\
$\mathrm{Hb} \times \mathrm{D}$ & $0.00177(0.00367)$ & $-0.000693^{* * *}(0.000184)$ \\
Age & $-0.0410^{* * *}(0.00431)$ & $0.00691(0.00553)$ \\
Age & $0.00125^{* * *}(0.000106)$ & $0.0367^{* * *}(0.00360)$ \\
Age & $-1.03 \times 10^{-5 * * *}\left(8.28 \times 10^{-7}\right)$ & $-0.000440^{* * *}\left(8.53 \times 10^{-5}\right)$ \\
$R^{2}$ & 0.052 & $1.38 \times 10^{-6 * *}\left(6.38 \times 10^{-7}\right)$ \\
\hline $\begin{array}{l}\text { Version } B \\
\text { Dependent variable: number of donation at- }\end{array}$ & Females & 0.084 \\
tempts within the next 18 months & & \\
\hline Mean (dependent variable) & 1.200 & Males \\
LHD & $0.00884(0.0570)$ & \\
Hb & $-0.00145^{* *}(0.000677)$ & $-0.00226^{* * *}(0.000629)$ \\
Hb $\times$ D & $0.0161^{*}(0.00849)$ & $0.0106(0.0148)$ \\
Age & $-0.0939^{* * *}(0.0155)$ & $0.0662^{* * *}(0.0137)$ \\
Age & & $-0.000424(0.000341)$ \\
Age & & \\
$R^{2}$ & $0.00295^{* * *}(0.000395)$ & $-1.50 \times 10^{-6}\left(2.65 \times 10^{-6}\right)$ \\
$F$ tests of instrument & $-2.43 \times 10^{-5 * * *}\left(3.17 \times 10^{-6}\right)$ & 0.090 \\
Observations & 0.066 & $1,190.67$ \\
\hline & $6,047.41$ & 101,650 \\
\hline
\end{tabular}

The effects of LHD on donor return were estimated using a linear probability model by 2SLS (for more detail, see equations A.2 and A.3 in the online suppl. material). In version A the outcome variable is the probability of making at least 1 donation attempt, and in version $B$ the outcome variable is the number of donation attempts within the next 18 months. All regressions additionally include 53 month fixed effects and control for blood types. Individual cluster robust SE are in parentheses. ${ }^{*} p<0.1 .{ }^{* *} p<0.05 .{ }^{* * *} p<0.01$.

attempt $(p<0.001)$ and of 0.00145 in the number of donation attempts $(p=0.033)$. Compared to $\mathrm{Hb}$ values above the cutoff, a 1-unit increase in the Hb value below the cutoff is related to an increase of 0.177 percentage points in the probability of making at least one donation attempt ( $p$ value: 0.630 ) and of 0.0161 in the number of donation attempts ( $p$ value: 0.058 ). For male donors, a 1 -unit increase in the $\mathrm{Hb}$ value above the cutoff is related to a decrease of 0.0693 percentage points in the probability of donating at least once $(p<0.001)$ and of 0.00226 in the number of donation attempts $(p<0.001)$. Compared to $\mathrm{Hb}$ values above the cutoff, a 1-unit increase in the $\mathrm{Hb}$ value below the cutoff is related to an increase of 0.691 percentage points in the probability of making at least 1 donation attempt $(p=0.211)$ and of 0.0106 in the number of donation attempts $(p=0.476)$.

The estimated age polynomials indicate that the relationship between age and donor return is inversely $\mathrm{U}$ shaped. In version B of the model, both female and male donors have the highest propensity to make donation at- tempts at the age of 60 years. Compared to a 60 -year old female donor, a 20 -year old female donor is predicted to make 0.63 fewer donation attempts, a 40 -year old female donor is predicted to make 0.33 fewer donation attempts, and a 70-year old female donor is predicted to make 0.19 fewer donation attempts. Compared to a 60 -year old male donor, a 20 -year old male donor is predicted to make 0.98 fewer donation attempts, a 40 -year old male donor is predicted to make 0.25 fewer donation attempts, and a 70year old male donor is predicted to make 0.08 fewer donation attempts. Qualitatively, the relationship is identical in version $\mathrm{A}$ of the model.

\section{Effects of the First versus Repeated LHD on Donor Return}

Table 3 shows the results of the second empirical model, differentiating between the effects of the first versus repeated LHD (see online suppl. Table S3 for first-stage regressions and online suppl. Fig. S1 for graphical illustrations). 
Table 3. Causal effect of repeated LHD on donor return

\begin{tabular}{|c|c|c|}
\hline \multicolumn{3}{|l|}{ Version A } \\
\hline $\begin{array}{l}\text { Dependent variable: probability of making at least } \\
1 \text { donation attempt within the next } 18 \text { months }\end{array}$ & Females & Males \\
\hline Mean (dependent variable) & 0.655 & 0.717 \\
\hline LHD & $-0.0216(0.0237)$ & $-0.0532(0.0360)$ \\
\hline $\mathrm{LHD} \times$ past $\mathrm{LHD}$ & $-0.135^{* * *}(0.0288)$ & $-0.133^{* * *}(0.0463)$ \\
\hline Past LHD & $0.120^{* * *}(0.00774)$ & $0.0798^{* * *}(0.00891)$ \\
\hline $\mathrm{Hb}$ & $-0.000471^{*}(0.000244)$ & $-0.000526^{* * *}(0.000185)$ \\
\hline $\mathrm{Hb} \times \mathrm{D}$ & $0.00131(0.00371)$ & $0.00614(0.00560)$ \\
\hline Age & $-0.0415^{* * *}(0.00427)$ & $0.0365^{* * *}(0.00359)$ \\
\hline $\mathrm{Age}^{2}$ & $0.00126^{* * *}(0.000105)$ & $-0.000436^{* * *}\left(8.51 \times 10^{-5}\right)$ \\
\hline $\mathrm{Age}^{3}$ & $-1.03 \times 10^{-5 * * *}\left(8.18 \times 10^{-7}\right)$ & $1.35 \times 10^{-6 * *}\left(6.37 \times 10^{-7}\right)$ \\
\hline$R^{2}$ & 0.055 & 0.084 \\
\hline $\mathrm{LHD}+\mathrm{LHD} \times$ past $\mathrm{LHD}=0$ & $2.30 \times 10^{-5}$ & 0.00179 \\
\hline \multicolumn{3}{|l|}{ Version $B$} \\
\hline $\begin{array}{l}\text { Dependent variable: number of donation attempts } \\
\text { within the next } 18 \text { months }\end{array}$ & Females & Males \\
\hline Mean (dependent variable) & 1.200 & 1.442 \\
\hline LHD & $0.0430(0.0568)$ & $-0.227^{* *}(0.0955)$ \\
\hline LHD $\times$ past $L H D$ & $-0.387^{* * *}(0.0738)$ & $-0.152(0.149)$ \\
\hline Past LHD & $0.411^{* * *}(0.0336)$ & $0.336^{* * *}(0.0495)$ \\
\hline $\mathrm{Hb}$ & $0.000126(0.000670)$ & $-0.00156^{* *}(0.000622)$ \\
\hline $\mathrm{Hb} \times \mathrm{D}$ & $0.0150 *(0.00857)$ & $0.0112(0.0150)$ \\
\hline Age & $-0.0956^{* * *}(0.0153)$ & $0.0654^{* * *}(0.0136)$ \\
\hline $\mathrm{Age}^{2}$ & $0.00297^{* * *}(0.000389)$ & $-0.000409(0.000338)$ \\
\hline $\mathrm{Age}^{3}$ & $-2.44 \times 10^{-5 * * *}\left(3.12 \times 10^{-6}\right)$ & $-1.63 \times 10^{-6}\left(2.63 \times 10^{-6}\right)$ \\
\hline$R^{2}$ & 0.073 & 0.092 \\
\hline $\mathrm{LHD}+\mathrm{LHD} \times$ past $\mathrm{LHD}=0$ & 0.000184 & 0.0346 \\
\hline$F$ tests of instrument & $1,826.76$ & 573.80 \\
\hline Observations & 72,025 & 101,650 \\
\hline
\end{tabular}

The effects of repeated LHD on donor return were estimated using a linear probability model by 2SLS (see equations A.4 and A.6 in the online suppl. material). In version A the outcome variable is the probability of making at least 1 donation attempt, and in version $B$ the outcome variable is the number of donation attempts within the next 18 months. All regressions additionally include 53 month fixed effects and control for blood types. Individual cluster robust SE in parentheses. ${ }^{*} p<0.1 .^{* *} p<0.05 .{ }^{* * *} p<0.01$.

When female donors experience their first LHD, their return rate is affected neither in terms of the probability of making at least 1 donation attempt $(p=0.362)$ nor in terms of the number of donation attempts within the next 18 months $(p=0.449)$. However, when they experience repeated LHD, their return rate drops significantly. They are 13.53 percentage points $(p<0.001)$ less likely to make at least 1 donation attempt within the first 18 months after a repeated LHD compared to the first LHD. They also make $0.387(\mathrm{p}<0.001)$ fewer donation attempts within the same period. Relating the estimated effect to the baseline number of donation attempts (online suppl. Table S1) shows that female donors make $0.387 / 1.200=32.25 \%$ fewer donation attempts after repeated LHD.
In contrast, male donors react to the first LHD. While the probability of making at least 1 donation attempt within 18 months of their first LHD drops only insignificantly by 5.32 percentage points $(p=0.139)$, they make 0.227 ( $p=0.018)$ fewer donation attempts over the same period. Relating this effect to the baseline number of donation attempts (online suppl. Table S4) shows that male donors make $0.227 / 1.442=15.74 \%$ fewer donation attempts after the first LHD. The difference in the estimated coefficients between male and female donors is insignificant in version $\mathrm{A}(p=0.503)$ but significant in version $\mathrm{B}(p=0.028)$ of the model.

When male donors experience repeated LHD, they are 13.30 percentage points $(p=0.004)$ less likely to make at least 1 donation attempt within 18 months compared to 
the first LHD. They also make 0.152 fewer donation attempts $(p=0.308)$ during the same period. Relating the estimated effect to the baseline number of donation attempts (online suppl. Table S1) shows that male donors donate an additional $0.152 / 1.442=0.54 \%$ less after repeated LHD. The estimated coefficients of the interaction terms do not differ significantly for female and male donors, either in version A $(p=0.974)$ or in version $\mathrm{B}(p=$ 0.210 ) of the model. The remaining coefficients have a similar effect as before and can be interpreted as in the first empirical model.

\section{Discussion}

This study estimates and quantifies the causal effects of LHD on donor return. After a first LHD, female donor return reacts neither in terms of the probability of making a donation attempt (point estimate: $-0.034 ; p=0.150$ ) nor in terms of the number of donation attempts (point estimate: $0.009 ; p=0.877$ ). In contrast, male donors are 6.07 percentage points $(p=0.095)$ less likely to make a donation attempt within the first 18 months after a first LHD and also make $0.221(p=0.024)$ fewer donation attempts within the same period.

However, when female donors experience repeated LHD, their return rate drops significantly. They are 13.53 percentage points $(p<0.001)$ less likely to make a donation attempt within the next 18 months and make 0.378 $(p<0.001)$ fewer donation attempts within the same period compared to the first LHD. When male donors experience repeated LHD, they are 13.30 percentage points $(p=0.004)$ less likely to make a donation attempt within 18 months and make 0.152 fewer donation attempts $(p=$ 0.308 ) during the same period compared to the first LHD.

In sum, male donors react to the first LHD whereas female donors respond only after repeated LHD. One reason for this pattern might be the fact that female donors are more likely to display temporarily insufficient $\mathrm{Hb}$ values, as the rule for LHD is stricter for female donors given their baseline $\mathrm{Hb}$ values and, at the same time, their $\mathrm{Hb}$ values fluctuate more due to the menstruation cycle. Consequently, LHD are more common for female donors and might be communicated in a less detrimental way.

Our contribution to the previous literature on donor return is mainly 2 -fold. First, we apply an RD design which allows us to quantify the causal effects of LHD on donor return. Previous studies have shown negative correlations between LHD and donor return; however, to what extent these negative effects were caused by the LHD per se rather than by unobserved factors remained previously unexplored. On the one hand, conditions, such as anemia, that lead to low Hb values and LHD could prevent donors from making future donations. On the other hand, conditions that lead to high $\mathrm{Hb}$ values, such as smoking [26] and dehydration [27], may also obstruct donor return. Therefore, it is crucial to filter out any unobserved factors that could confound the results. The RD design allows us to estimate the causal effects of LHD that are not driven by those unobserved factors. As a result, our estimates are smaller in magnitude comparing to previous studies [17-21] that omitted those unobserved factors underlying the LHD. Second, we not only estimate the causal effects of LHD overall but also discriminate between the causal effects of the first versus repeated LHD. The finding that female donors react much more strongly to repeated LHD than to the first LHD is, to our knowledge, novel as well.

More broadly, since voluntary blood donation is a textbook example of prosocial behavior, our results are in line with the economic literature on excuse-driven responses in prosocial behavior $[10,11]$, when deferred donors use LHD as excuses for not returning. Our results are also consistent with the psychology literature on the negative effects of social exclusion [13-16] on prosocial behavior, when deferred donors perceive LHD as social exclusion.

The results are highly policy relevant. First of all, the results indicate that blood transfusion services should try to minimize unnecessary LHD due to false measurement. It might be worthwhile to use more accurate measurement devices to avoid such unnecessary LHD, even if they entail higher costs and longer measurement times.

Moreover, communicating LHD to deferred donors should be considered a delicate matter. Transfusion services could staff additional personnel to spend more time on: (1) comforting deferred donors and explaining to them that insufficient $\mathrm{Hb}$ values are often temporary, (2) preparing a take-home message that, for example, suggests an iron- and vitamin-rich diet over the next weeks, and (3) drafting a more delicate invitation letter for those who were previously deferred.

Additionally, the BTSRC could change the uniform invitation procedure and differentiate the causes of the low $\mathrm{Hb}$ values. Currently, deferred donors are recommended to wait 3 months before making the next donation attempt. The BTSRC sends the same invitation letter after the same waiting period to deferred and nondeferred donors. While this waiting time is necessary for deferred donors with a chronic iron deficiency and severe diseases, it is probably unnecessarily long for deferred donors who simply have temporary low $\mathrm{Hb}$ values caused by other conditions. If the BTSRC could identify and differentiate the causes of the low Hb values, it could recommend more specific waiting times and encourage 
deferred donors with good health conditions to return sooner.

Another solution to avoid the frustration caused by LHD may be to allow donors with an $\mathrm{Hb}$ value just below the cutoff to donate nevertheless and, subsequently, discard their blood transfusions as they do not meet the required quality standards. However, the benefit of this solution would have to be carefully assessed against its cost and reputational risk.

Testing the effectiveness of the above solutions also opens up an avenue for future research. Our methodology should be applicable in such tests.

This study has some limitations. First, we only provide evidence for the effects of LHD, but remain silent about the effects of STTD due to other reasons. Second, our identification comes from donors with $\mathrm{Hb}$ values close to the cutoff. Whether the results are valid for donors with $\mathrm{Hb}$ values that are far away from the cutoff remains unclear. However, deferrals of donors close to the cutoff are the most relevant STTD, as donors far above or far below the cutoff are rarely deferred or rarely eligible.

\section{Statement of Ethics}

All of the subjects gave their informed consent in written form. There were no animals involved. The study protocol was approved on May 27, 2016, by the institute's ethics committee on human research (Committee on Ethics in Research, HEC Lausanne, University of Lausanne).

\section{Disclosure Statement}

The authors declare that they have no conflict of interests relevant to this paper.

\section{Funding Sources}

The study was partly funded by the Swiss National Science Foundation (grant No. 141767).

\section{Author Contributions}

A.B., L.G., S.H., and L.J. analyzed the data and wrote this paper. A.B., L.G., S.H., L.J., A.M., and B.M.F. designed this study and reviewed this paper. A.R. and R.B. helped to collect the data.

\section{References}

1 Cable RG. Hemoglobin determination in blood donors. Transfus Med Rev. 1995 Apr; 9(2):131-44.

2 James V, Jones KF, Turner EM, Sokol RJ. Statistical analysis of inappropriate results from current $\mathrm{Hb}$ screening methods for blood donors. Transfusion. 2003 Mar;43(3):400-4.

3 Sawant RB, Bharucha ZS, Rajadhyaksha SB. Evaluation of hemoglobin of blood donors deferred by the copper sulphate method for hemoglobin estimation. Transfus Apheresis Sci. 2007 Apr;36(2):143-8.

4 Miller RD, Ward TA, Shiboski SC, Cohen $\mathrm{NH}$. A comparison of three methods of hemoglobin monitoring in patients undergoing spine surgery. Anesth Analg. 2011 Apr; 112(4):858-63

5 Goette L, Stutzer A. Blood Donations and Incentives: Evidence from a Field Experiment. Discussion Paper No. 3580. Bonn: IZA; 2008.

6 Goette L, Stutzer A, Yavuzcan G, Frey BM. Free cholesterol testing as a motivation device in blood donations: evidence from field experiments. Transfusion. 2009 Mar;49(3):52431.

7 Goette L, Stutzer A, Frey BM. Prosocial motivation and blood donations: A survey of the empirical literature. Transfus Med Hemother. 2010 Jun;37(3):149-54.

8 Lacetera N, Macis M. Do all material incentives for pro-social activities backfire? The response to cash and non-cash incentives for blood donations. J Econ Psychol. 2010;31(4): 738-48.

9 Mast AE, Schlumpf KS, Wright DJ, Custer B, Spencer B, Murphy EL, et al.; NHLBI Retrovirus Epidemiology Donor Study-II. Demographic correlates of low hemoglobin deferral among prospective whole blood donors. Transfusion. 2010 Aug;50(8):1794-802.

10 Haisley EC, Weber RA; Emily C Haisley and Roberto A Weber. Self-serving interpretations of ambiguity in other-regarding behavior. Games Econ Behav. 2010;68(2):614-25.

11 Exley CL. Excusing selfishness in charitable giving: the role of risk. Rev Econ Stud. 2016; 83(2):587-628.

12 Baumeister RF, Leary MR. The need to belong: desire for interpersonal attachments as a fundamental human motivation. Psychol Bull. 1995 May;117(3):497-529.

13 Nezlek JB, Kowalski RM, Leary MR, Blevins T, Holgate S. Personality moderators of reactions to interpersonal rejection: depression and trait self-esteem. Pers Soc Psychol Bull. 1997;23(12):1235-44.

14 Eisenberger NI, Lieberman MD, Williams KD. Does rejection hurt? An FMRI study of social exclusion. Science. 2003 Oct;302(5643): 290-2.

15 Twenge JM, Baumeister RF, DeWall CN, Ciarocco NJ, Bartels JM. Social exclusion decreases prosocial behavior. J Pers Soc Psychol. 2007 Jan;92(1):56-66.

16 Gerber J, Wheeler L. On being rejected a meta-analysis of experimental research on rejection. Perspect Psychol Sci. 2009 Sep;4(5):46888.

17 Halperin D, Baetens J, Newman B. The effect of short-term, temporary deferral on future blood donation. Transfusion. 1998 Feb;38(2): 181-3.

18 Custer B, Chinn A, Hirschler NV, Busch MP, Murphy EL. The consequences of temporary deferral on future whole blood donation. Transfusion. 2007 Aug;47(8):1514-23.
19 Zou S, Musavi F, Notari EP, Rios JA, TrouernTrend J, Fang CT. Donor deferral and resulting donor loss at the American Red Cross Blood Services, 2001 through 2006. Transfusion. 2008 Dec;48(12):2531-9.

20 Custer B, Schlumpf KS, Wright D, Simon TL, Wilkinson S, Ness PM; NHLBI Retrovirus Epidemiology Donor Study-II. Donor return after temporary deferral. Transfusion. 2011 Jun;51(6):1188-96.

21 Hillgrove T, Moore V, Doherty K, Ryan P. The impact of temporary deferral due to low hemoglobin: future return, time to return, and frequency of subsequent donation. Transfusion. 2011 Mar;51(3):539-47.

22 Downey G, Mougios V, Ayduk O, London $\mathrm{BE}$, Shoda Y. Rejection sensitivity and the defensive motivational system: insights from the startle response to rejection cues. Psychol Sci. 2004 Oct;15(10):668-73.

23 Peterson C. Steven F Maier, and Martin EP Seligman. Learned helplessness: A theory for the age of personal control. USA: Oxford University Press; 1993.

24 von Schenck H, Falkensson M, Lundberg B. Evaluation of "HemoCue," a new device for determining hemoglobin. Clin Chem. 1986 Mar;32(3):526-9.

25 Angrist JD, Pischke JS. Mostly Harmless Econometrics: An Empiricist's Companion. New Jersey: Princeton University Press; 2008.

26 Leifert JA. Anaemia and cigarette smoking. Int J Lab Hematol. 2008 Jun;30(3):177-84.

27 Henny H. Billett. Hemoglobin and Hematocrit. In: Walker HK, Hall WD, Hurst JW, editors. Clinical Methods: The History, Physical, and Laboratory Examinations. 3rd ed. Boston: Butterworths; 1990. 